\title{
STUDIES ON UROBILIN PHYSIOLOGY AND PATHOLOGY.
}

\author{
III. Absorption of Pigments of Biliary Derivation from \\ THE INTESTINE.
}

Bx PHILIP D. MCMASTER, M.D., AND ROBERT ELMAN, M.D.

(From the Laboratories of The Rockefeller Institute for Medical Research.)

(Received for publication, March 10, 1925.)

INTRODUCTION.

Evidence for an enterohepatic circulation of bile pigment has been offered in a previous communication from this laboratory. ${ }^{1}$ It is our present purpose to record experiments on the absorption from the gastrointestinal tract, not only of bilirubin but of urobilin. Many new findings which indirectly testify to the absorption of these pigments of biliary derivation will also be described.

In the course of studies already reported, ${ }^{2}$ dogs losing the total bile as the result of common duct intubation, and in consequence urobilin-free-the bile, feces, and urine containing none of the pigment-were fed either their own bile or urobilin-free bile from similar urobilin-free dogs. All the animals so fed exhibited a great increase of the bilirubin of the bile and urobilin appeared in it within 48 hours. From this fact and from others it became evident that urobilin appears in the normal bile only when bile pigment in some form was allowed to reach the intestinal tract. The evidence pointed to the absorption from the intestine either of the pigment or of substances out of which it might be formed. A closer study of the problem of this absorption has been undertaken. For the purpose pure bilirubin and urobilin have been used and the total bile collected from day to day in a sterile condition according to a special method. ${ }^{3}$

1 Broun, G. O., McMaster, P. D., and Rous, P., J. Exp. Med., 1923, xxxvii, 699.

McMaster, P. D., and Elman, R., J. Exp. Med., 1925, xli, 513.

' Rous, P., and McMaster, P. D., J. Exp. Med., 1923, xxxvii, 11. 
PREVIOUS LITERATURE.

The majority of previous workers have assumed, on indirect evidence, that bile pigment is absorbed from the gastrointestinal tract and resecreted by the liver. The work of Wilbur and Addis ${ }^{\mathbf{s}}$ and of Addis ${ }^{5}$ furthered this view while summarizing the general opinion of investigators up to that time. Recently doubt has been cast upon it by authors ${ }^{6,7}$ who pointed out that proof of any absorption of bilirubin and urobilin is wanting, - an assertion made, be it said, prior to the publication of papers from this laboratory, which go far toward supplying such proof., In one of the papers referred to, Broun, McMaster, and Rous ${ }^{1}$ showed that feedings of dog bile to dogs with intubated common duct caused within the next 24 to 48 hours great increases in the bilirubin output by the liver. Furthermore feedings of sheep bile containing cholohematin were followed by the appearance of this pigment in the bile secreted in the next 24 hours. Caution was required in the interpretation of the findings with cholohematin since it has been shown that the substance is not a normal constituent of sheep or dog bile. However, the increased output of bilirubin following feedings of dog bile admit of no such objection. Recently it has been demonstrated that complete bile deprivation causes urobilin to disappear from the body, and that feedings of bile to such animals are followed by the reappearance of this pigment in the bile and feces. ${ }^{2}$

To these experimental findings may be added certain clinical observations of value. The crucial experiment of Friedrich Müller stands out amongst them. ${ }^{8}$ To a patient heavily jaundiced, in whose colorless stools and dark urine there was no trace of urobilin, he fed urobilin-free pig bile. In 2 days the pigment appeared in the feces, and a day later in the urine. This experiment has since been confrmed by several workers. ${ }^{9,10}$ Recently Meyer and Heinelt ${ }^{11}$ injected bile directly into the intestine of human patients through a duodenal tube and found an increase in bilirubinemia, above that which is now recognized to be normal to man.

\section{Method.}

For the present study healthy dogs were used, permanently intubated, some for the collection of total bile, some for samples of bile drained from a single liver lobe only, and others with tubes so arranged that either the total bile could

${ }^{4}$ Wilbur, R. L., and Addis, T., Arch. Int. Med., 1914, xiii, 235.

${ }^{5}$ Addis, T., Arch. Int. Med., 1915, xv, 413.

${ }^{6}$ Hooper, C. W., and Whipple, G. H., Am.J. Physiol., 1916-17, xlii, 264.

${ }^{7}$ Whipple, G. H., Physiol. Rev., 1922, ii, 440.

${ }^{8}$ Müller, F., Verhandl. Congr. inn. Med., 1892, xi, 118.

- Fromholdt, G., and Nersessoff, N., Z. exp. Path. u. Pharmakol., 1912, xi, 400.

${ }^{10}$ Fischer, H., and Meyer-Betz, F., Munch. med. Woch., 1912, lix, 799.

${ }^{11}$ Meyer, E. C., and Heinelt, H., Deutsch. Arch. klin. Med., 1923, cxlii, 94. 
be obtained in the collecting apparatus, or else it could flow back into the normal channels and so to the intestine. ${ }^{2}$ The methods for bile collection, in 24 hour periods, and for analyses of the bile specimens have been described in previous papers, ${ }^{2,12}$ together with the management of the animals. Only the data from animals with sterile bile will be considered in the analysis of results.

\section{The Effect of Bile Loss on the Liver Output of Bile Pigments.}

Studies of the bilirubin output into the bile were carried out upon nine dogs with the "altercursive biliary intubation,"13 the same animals used in a previous study. ${ }^{2}$ Following operation such an animal could be maintained either as an essentially normal dog, or as one deprived of the total bile; and the conditions could be alternated. After intubation the bile was regularly allowed to flow into the intestine for 1 or 2 weeks, that is to say until the effects of laparotomy had disappeared and the animal was in excellent condition. Previous work on animals from which all of the bile was collected had taught us that the output of bilirubin becomes practically constant within six to ten days after operation. ${ }^{14}$ We began to collect the bile from dogs with "altercursive biliary intubation" after this condition of affairs had presumably established itself. It will be seen in the charts (Text-figs. 1 and 9 and Text-fig. 8 of the previous paper ${ }^{2}$ ) that instead of being uniform, the amount of pigment eliminated in the bile was greater in the first few 24 hour periods of collection than at any time later, and progressively declined, becoming approximately uniform only after 6 or 8 days had elapsed. The bile contained urobilin during the first 2 or 3 days of collection, but failed to show its presence thereafter (Text-fig. 9). When the bile was again allowed to enter the intestine for a week or more, and then again diverted to the collecting apparatus, the same "toboggan slide" effect in bilirubin elimination was observed.

These results corroborate in a striking way our previous finding,

12 Elman, R., and McMaster, P. D., J. Exp. Med., 1925, xli, 503.

13 "Altercursive biliary intubation," a term coined for convenience of expression in the previous paper, ${ }^{2}$ to describe a type of intubation of the bile ducts, by which the flow of secretion could follow one of two courses, either that to the collecting balloon, or back into the common duct and so to the intestine.

14 McMaster, P. D., Broun, G. O., and Rous, P., J. Exp.Med., 1923, xxxvii, 395. 
that the occurrence of urobilin in the bile is dependent on the presence of some of this secretion in the intestine. They confirm as well the observations of Broun, McMaster, and Rous, ${ }^{1}$ that the bilirubin falls off when bile is permanently diverted from the gut but can be increased again by bile feedings.

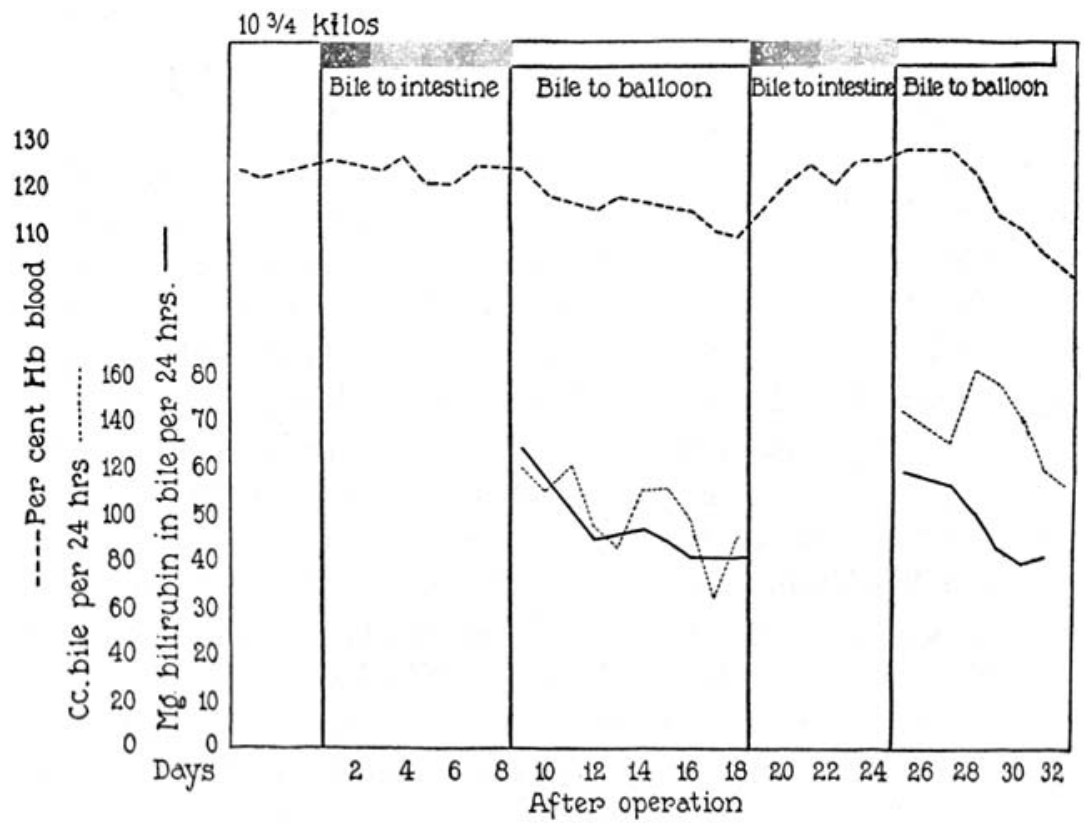

Text-Fig. 1. Effect of bile loss on the output of bile pigment.

For 8 days after operation the bile of Dog F had been allowed to flow into the intestine. When now the secretion was diverted, and collected at 24 hour intervals, the bilirubin output fell off rapidly, tending to reach a level only after the lapse of 6 days. When the bile had again been allowed to flow into the intestine, and again been diverted to the collecting apparatus, the same changes in the bilirubin output occurred. No urobilin estimations were made.

\section{Results of Feeding Bile Containing Bilirubin and Urobilin.}

The bilirubin and urobilin outputs were followed in eight dogs with intubated common duct during 10 to 30 days following intubation. At the end of this time the animals were in excellent condition, and were excreting a constant amount of bilirubin daily into the bile. 
The diet was a uniform one of $1,050 \mathrm{gm}$. of bread wet with milk mixed with $450 \mathrm{gm}$. of lean meat for an animal of about 12 kilos.

They were now fed, by gavage, 100 to $190 \mathrm{cc}$. of their own bile or that from other intubated animals-the dosage representing in each case approximately the daily output of bile pigment by the animal. This bile was urobilin-free. The amount of bilirubin administered in it was determined by the method used in quantitating the daily specimens. In each animal so fed a great increase in the bilirubin output ensued during the next 24 hours. Text-fig. 2 shows this for three animals. Prior to the feedings urobilin had not been found in the bile for many days. It appeared in each case on the day following the feeding and disappeared 1 or 2 days later. No protocols will be given, since the findings merely confirm those of the previous work. ${ }^{1,2}$

Far more striking are the findings when bile feedings are continued for days. Text-fig. 3 shows the sequence of events in a $16 \frac{3}{4}$ kilo animal from the time of intubation of the common duct to the termination of the observations. For some days after the operation for the collection of the bile its bilirubin output decreased then reaching a fairly uniform level. The fall was greater and more prolonged than that usually seen. By the 4th day after intubation urobilin had disappeared from the bile and the stools had become acholic.

On the 25th day of the experiment, 21 days after the animal had become urobilin-free, bile feedings were commenced, by gavage, and continued for 10 days. The material used, sterile and devoid of urobilin, was derived from another intubated animal. In addition to the noteworthy increase in the bilirubin output caused by the feedings, a phenomenon sufficiently described in previous papers, ${ }^{1,2}$ there was a reappearance of urobilin in the bile of the 24 hour period immediately after the first bile administration, and 2 days later in the feces. Both bile and feces continued to contain the pigment as long as bile was fed. Thereafter the output of bilirubin fell almost at once to its former level and the urobilin disappeared. Three of our dogs were repeatedly fed bile in this way, with similar results in each case.

In further experiments bile was given to animals intubated for the 
collection of a small part of the bile only. These, too, showed an increased bilirubin elimination after feedings of bile by mouth. Text-fig. 4 shows this effect of a single bile feeding to a dog losing but 28 per cent of its bile into the collecting apparatus. The animal was in excellent condition and had been gaining weight steadily since intubation. Urobilin had been constantly present in the bile samples.

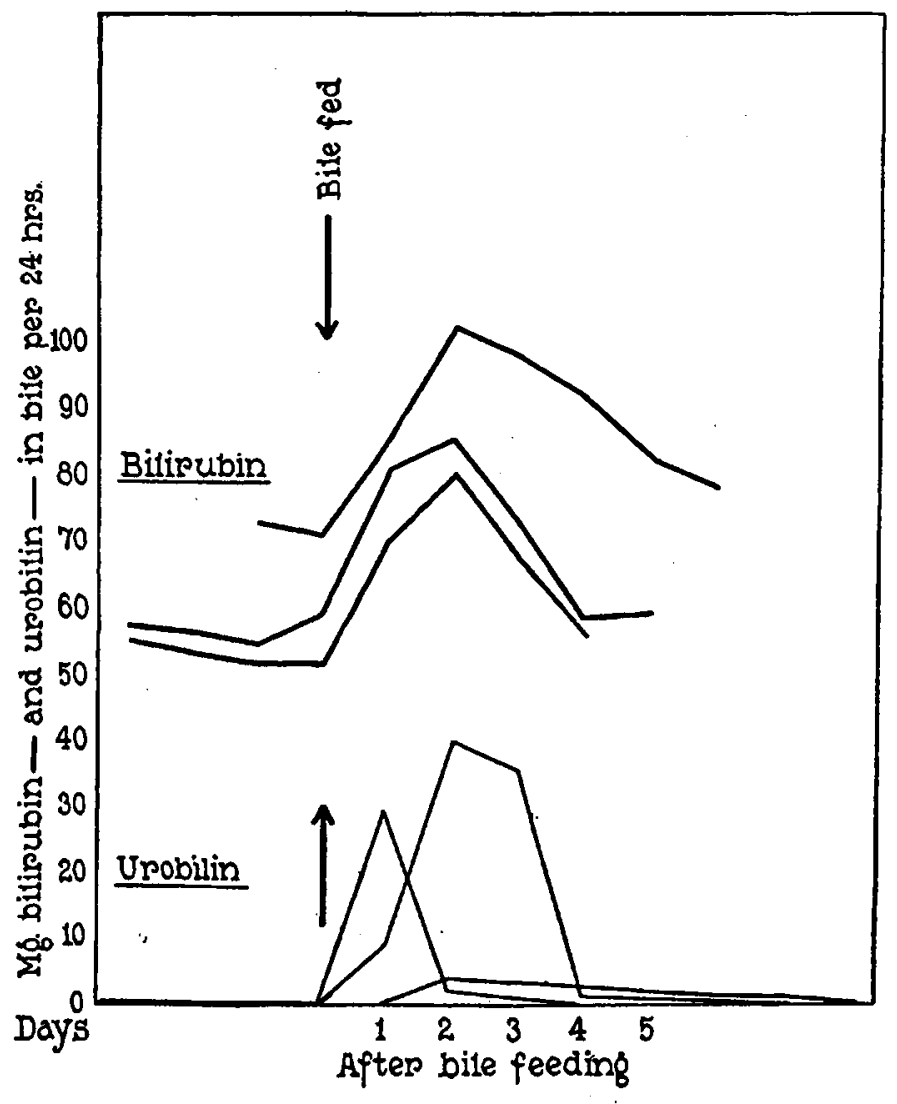

TEXT-Frg. 2. The increased output of bile pigments after a single bile feeding. The result of bile feedings to three animals with intubated common duct. The bilirubin output in each of these animals was practically uniform before the feedings were given. The bile was urobilin-free, but the pigment appeared in it promptly after the feeding and persisted there for several days. There was synchronous rise in the bilirubin output. 


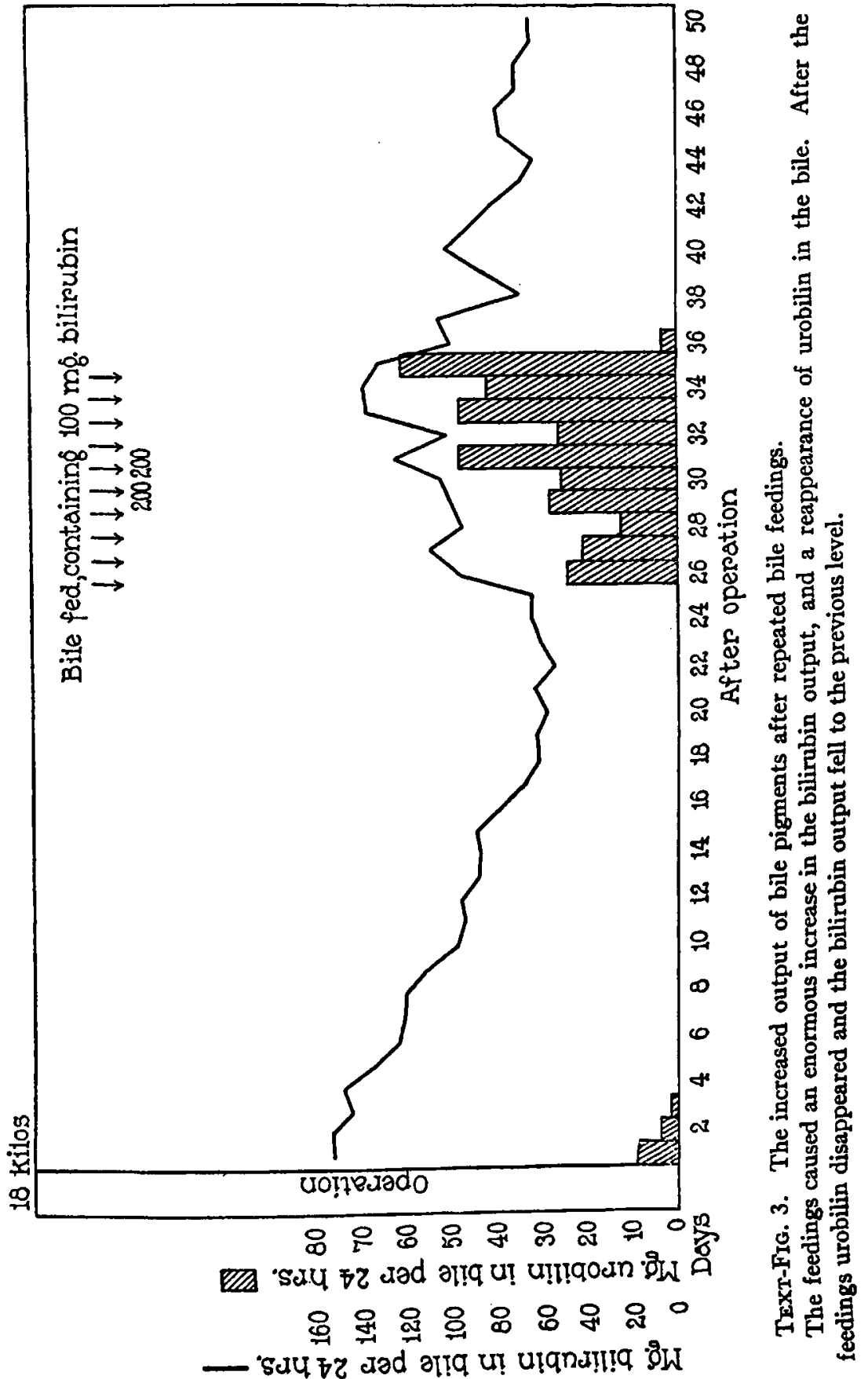


Dog C, 11 1/2 kilos, male. Under ether anesthesia cannulation of the duct to the left lateral lobe of the liver only. This lobe was determined at autopsy to be 28 per cent of the total liver. The animal was in excellent condition, on the standard mixed diet.

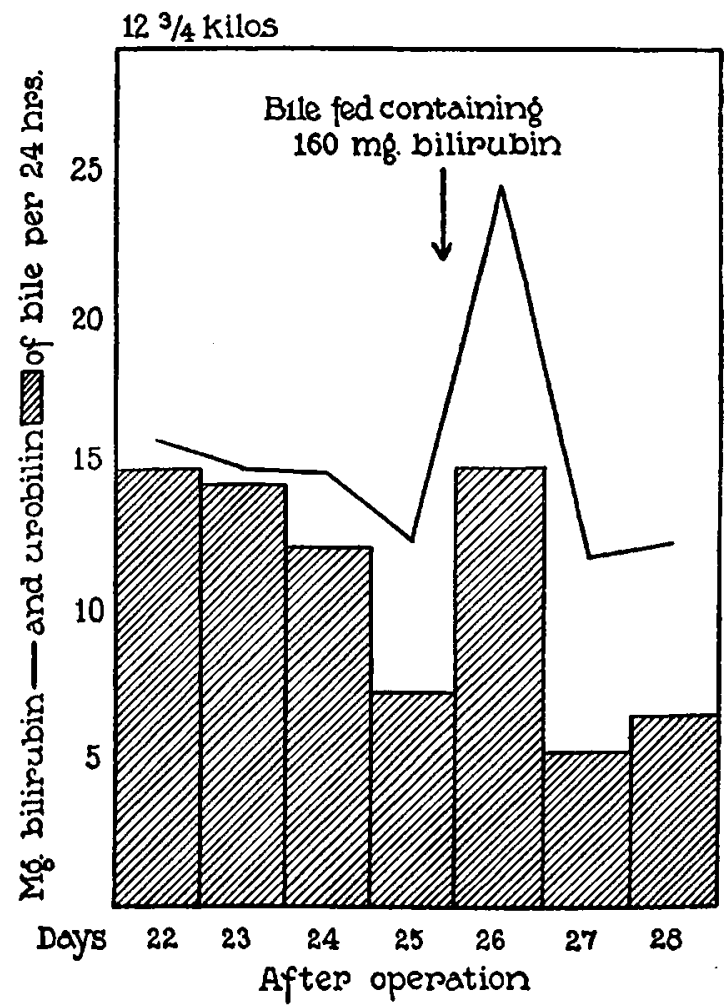

TEXT-Fig. 4. The increased output of bilirubin after feeding bile to an animal deprived of only a small part of the secretion.

It will be seen that on the day after a bile feeding involving $160 \mathrm{mg}$. of bilirubin there was an increase of more than 75 per cent in the output of this pigment. 2 days later the output had dropped again to the previous level.

26 days later the animal had gained 1 kilo in weight and was putting out sterile bile with a quite uniform bilirubin content, having $14.7,14.75$, and $12.18 \mathrm{mg}$. of bilirubin daily, from the 24th to the 25th days, in the 28 per cent sample of the secretion, during each 24 hour period. As in all animals so intubated, the bile and stools contained urobilin. On the 26th day $190 \mathrm{cc}$. of sterile urobilin-free bile, containing $160 \mathrm{mg}$. of bilirubin, derived from a dog with intubated common 
duct, was fed by mouth. As Text-fig. 4 shows a rise in the bilirubin climination in the bile sample from 12.18 to $24.25 \mathrm{mg}$. occurred in the following 24 hours; with a fall in the next period to the usual level, $-12.1 \mathrm{mg}$.

It might be argued that the increased bile pigment output after bile feedings in animals losing all of their bile might be due to a betterment in digestive or other conditions. But this explanation can scarcely be invoked in instances such as the foregoing. There were three such in two different animals.

\section{The Effect of Feeding Bile Salts.}

Feeding of whole bile to intubated dogs brings about not only an increase in the bilirubin output, but a great one in the quantity of bile, owing, of course, to the cholagogue action of the bile salts. When these latter fail to reach the intestine, as during bile collection by the "altercursive" method, the bile volume (Text-fig. 1) undergoes a gradual decrease. The increase in bilirubin eliminated after bile feeding is not proportional to the greater volume of bile put out. That bile volume and pigment quantity bear no relation to each other has already been shown.1,15

It seemed important to test the possibility whether the giving of bile salts by mouth might not induce the observed increase in bilirubin by a destruction of red cells. For bile salts exert a hemolytic action in vitro, though only to a marked degree when scrum is absent. Accordingly, four dogs with intubated common duct, and two with a "sampling intubation," all with a uniform daily output of bilirubin, were given varying amounts of sodium glycocholate by gavage. The same ration as previously was also provided. The outcome of the experiment, in two typical instances, is shown in Text-fig. 5, $a$ and $b$. The bile salts produced a strong cholagogue effect without altering the bilirubin output of the 24 hour sample.

\section{The Preparation of Purified Bile Pigments.}

It is evident that one cannot ascribe the increased output of bilirubin after feedings of whole bile to the action of the bile salts ingested

${ }^{15}$ McMaster, P. D., J. Exp. Med., 1924, xl, 25. 

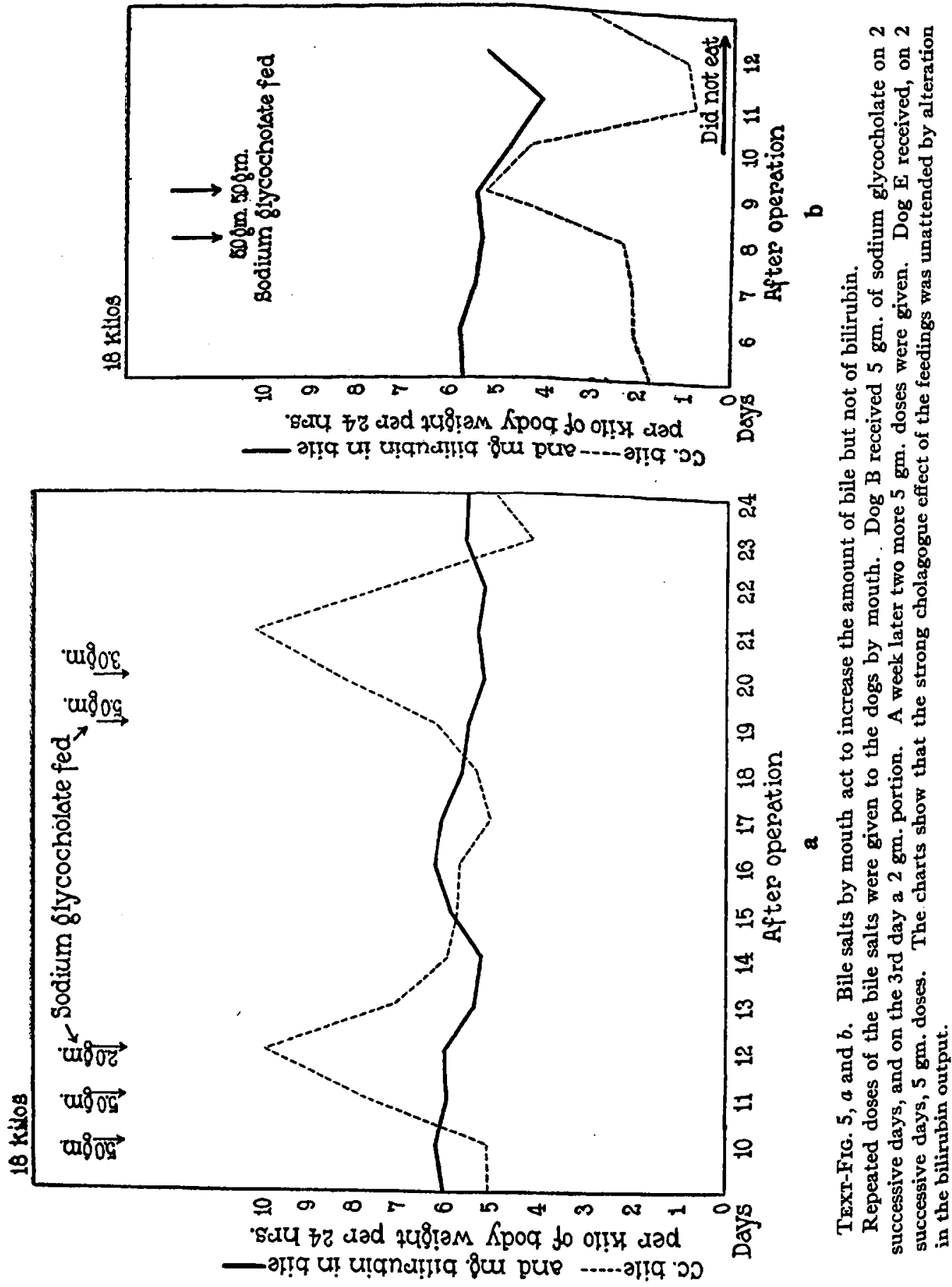
therewith. Like the large initial bilirubin output in animals with an "altercursive intubation," and like the relatively great output of animals with a mere "sampling intubation," the phenomenon must be due to some other constituent of the bile reaching the intestine. The explanation that this constituent is the bile pigment itself, which is in part reabsorbed, and resecreted, would, in view of present knowledge, seem the rational one. We have proceeded to test it.

Purified bilirubin and urobilin, were prepared and administered to intubated animals.

Preparation of Urobilin.-The method of preparation of urobilin from dog feces has already been described in another paper. ${ }^{12}$

Preparation of Pure Bilirubin from Dog Bile. - In all, about 10 liters of dog bile from the collecting balloons of animals with intubated common duct were utilized. As fast as a few $100 \mathrm{cc}$. had been assembled on ice it was used. Each lot was precipitated within 2 days so that little or no transformation of bilirubin to biliverdin would take place in it.

To each $100 \mathrm{cc}$. of fresh bile used, $10 \mathrm{cc}$. of 20 per cent $\mathrm{NaOH}$ was added and the mixture was brought to a boil. To the hot mixture, glacial acetic acid was added until precipitation was complete; 10 to $12 \mathrm{cc}$. usually giving the best results. Two precautions were found necessary for successful extraction of bilirubin, the use of fresh bile only, containing but little biliverdin, and boiling in a closely covered container, under circumstances making for the exclusion of air. The precipitate was collected on a filter paper and washed with water until the filtrate appeared clear and proved acid-free. Precipitate and filter paper were next removed to an extraction thimble in a Soxhlet apparatus, and extracted with alcohol until the latter came away clear. The alcohol was then replaced by chloroform, and the extraction repeated, the chloroform extract filtered, the excess of chloroform digtilled off and recovered, and the remaining filtrate evaporated to dryness in a current of air. This dry residue was washed again with alcohol, the fluid poured off, washing done with ether, which was in turn poured off; and washings with alcohol and with ether alternated as long as anything came away. The residue was then taken up in chloroform again, filtered, and the process of purifying repeated until only crystalline bilirubin remained.

Neither the bilirubin nor the urobilin showed any traces of bile salts by Hay's or Pettenkofer's test. The color intensity of solutions of the bilirubin, on the addition of acid alcohol, proved to be slightly stronger than that given by a Schuchardt preparation from ox bile. For the comparison a Duboscq colorimeter was used.

\section{The Effects of Feeding Purified Bilirubin.}

Pure bilirubin was given to eight dogs, three with intubated common duct, one with a "sampling intubation," and four with the "altercursive intubation." 
In general, $100 \mathrm{mg}$. were given, approximately the amount lost daily in the bile by most of the animals. It was dissolved before administration by the addition to each $100 \mathrm{mg}$. of $30.0 \mathrm{cc}$. of distilled water containing $1 \mathrm{cc}$. of 20 per cent $\mathrm{NaOH}$, Once in solution more water could be added without any falling out of pigment, and the volume was thus made up to that of the daily secretion of bile of the animal to receive the dose. Colorimetric determinations of the bilirubin, made by the same method as the quantitations of the daily bile samples, were found to check closely with the values obtained gravimetrically. Just before the solution was

given it was rendered neutral or very faintly acid by the addition of $\frac{\mathrm{N}}{10}$ hydrochloric acid, not enough being added to produce a change from bilirubin to biliverdin. To the animals with a common duct or a "sampling intubation" the solution was fed in divided doses, several hours apart, by stomach tube. It was injected in divided doses through the efferent tube into the common duct of those with the "altercursive intubation."

In every case the result was the same; great increases in the bilirubin output in the bile followed the feedings. Text-figs. 6 and 7 illustrate the results of bilirubin feedings by mouth to animals losing all of the bile. It will be seen that immediately after a single feeding of bilirubin the output of this pigment underwent an abrupt increase and did not return to the previous amount for several days. At the maximum there was a 65 per cent increase in the 24 hour specimen (see Text-fig. 6). Urobilin reappeared in the bile following the bilirubin gavage and disappeared again only after the course of several days. With all this there was no marked change in the bile quantity. Therein the findings differ notably from those obtained by whole bile feedings, in which the cholagogue action of bile salts is apparent.

Like findings were obtained in Dog $\mathrm{C}$, intubated for the collection of bile samples only and losing 28 per cent of the total secretion in this way, while the remainder passed as usual into the intestine (Text-fig. 8).

\section{Effects of the Introduction of Purified Bilirubin into the Duodenum.}

In the foregoing experiments the possibility of a direct gastric absorption of pigment, or of a gastric influence upon the bilirubin solution, rendering it capable of absorption by the intestine, could not be ruled out. To test these possibilities bilirubin in solution was instilled directly into the duodenum of dogs through the efferent 


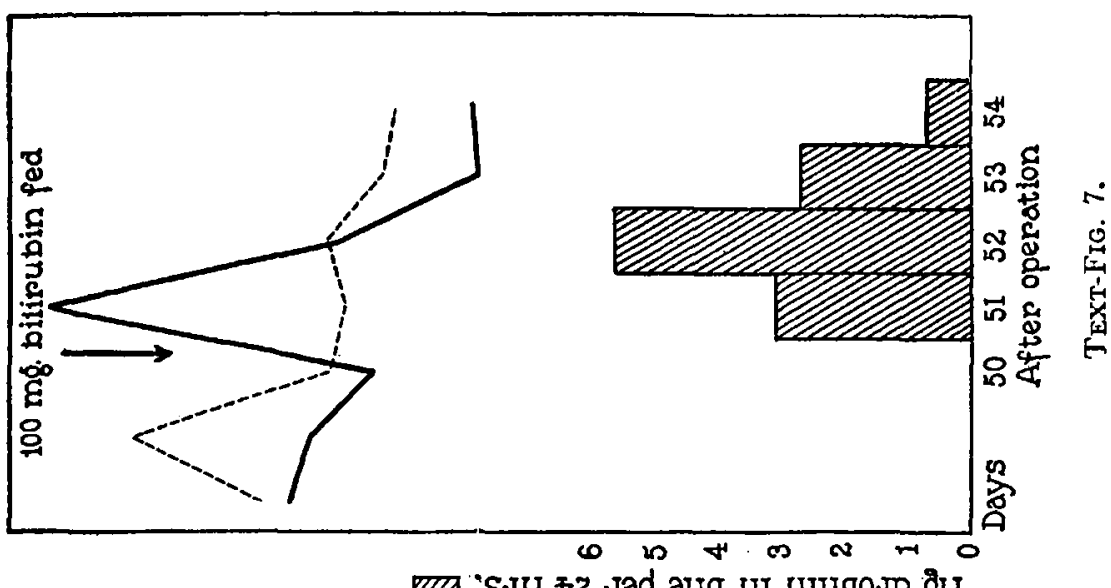

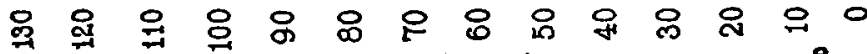

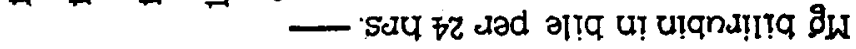

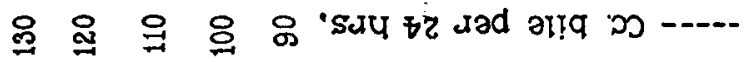

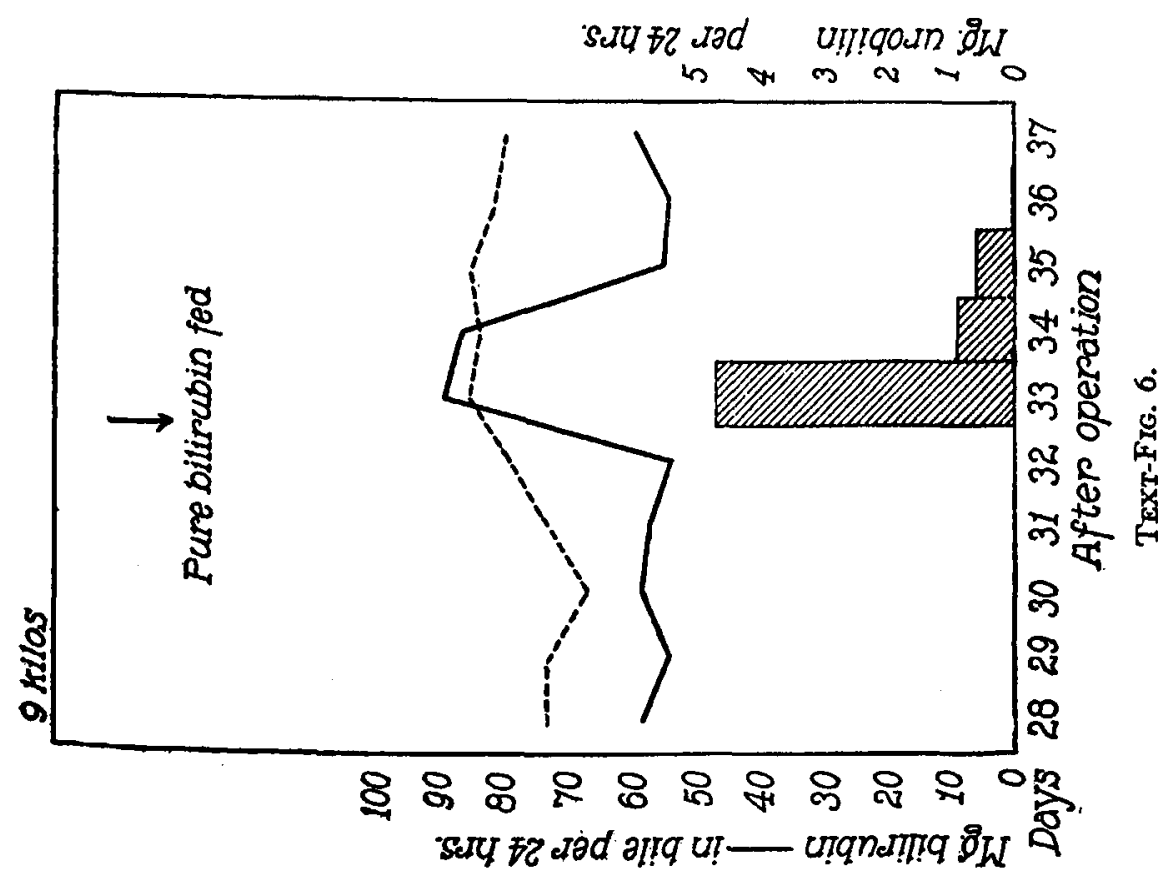

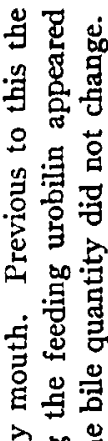

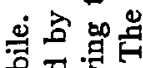

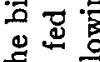
ฐ 范的事

코.

so 䒴 용ㅇㅇ 졀 명 屍完 8 㟧 更 몽. द्?

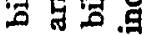
$\overrightarrow{8}$ 눙

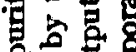
68

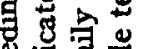
青完

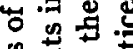

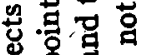
舁 过

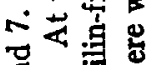
腾 的要守

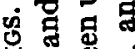
田 嵌品 臽号焉

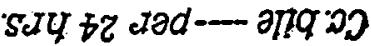


tube of the "altercursive intubation" apparatus, which is the one that connects with a cannula inserted into the lower portion of the common duct.2 Both before and after the injection the total hepatic bile. was collected as usual through the afferent tube, which connected another cannula, inserted into the upper part of the common duct, with the collecting balloon. Text-fig. 9 depicts the findings in an animal to which bilirubin was adminstered at widely separate intervals. A brief description of the experiment follows.

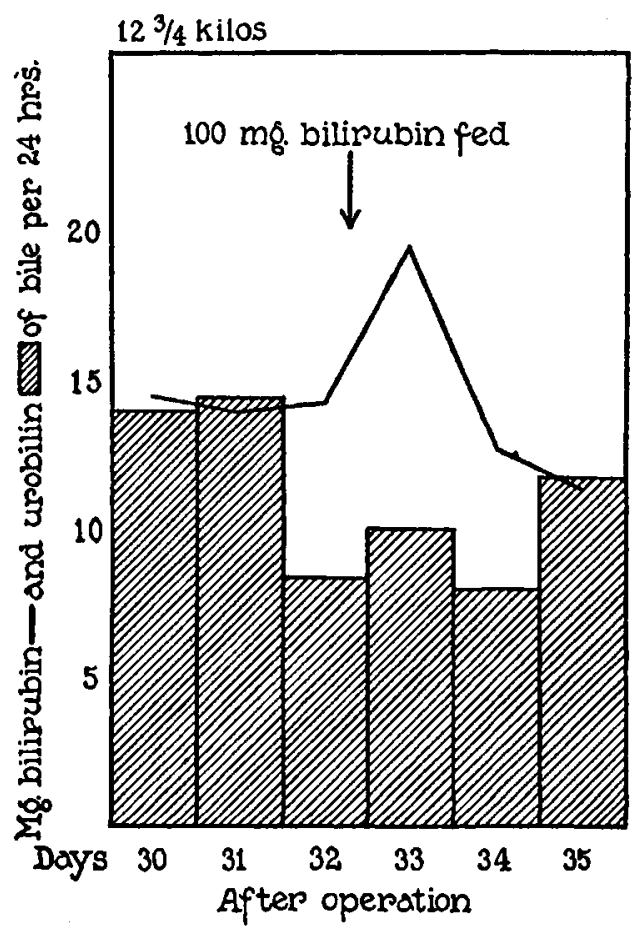

TEXT-FIG. 8. Effect of feeding purified bilirubin to an animal with a "sampling intubation."

The actual alterations observed in the bilirubin output in the bile sample have been charted, not the calculated changes in total output, which were much more considerable. It will be seen that the feeding of pigment was responsible for a notable increase in the output of it. Urobilin was present in the sample bile throughout the period of observation as would follow from the circumstances of the case. The observed variations in the amount of this pigment fell within the ordinary limits. 
Dog $D$, male, 12 kilos. During the first 5 days after intubation the bile was allowed to flow into the intestine. The amount of urobilin in the stools of this period was considerable. It fell off rapidly, though, and disappeared after bile collection had been begun. In the bile first collected there was far more bilirubin than later, and the day-to-day quantitation yielded the usual "toboggan slide" curve. When a level appeared to have been reached, the content of the bile being $66.6,67.5$, and $72.3 \mathrm{mg}$. for three successive 24 hour periods, $100 \mathrm{mg}$. of

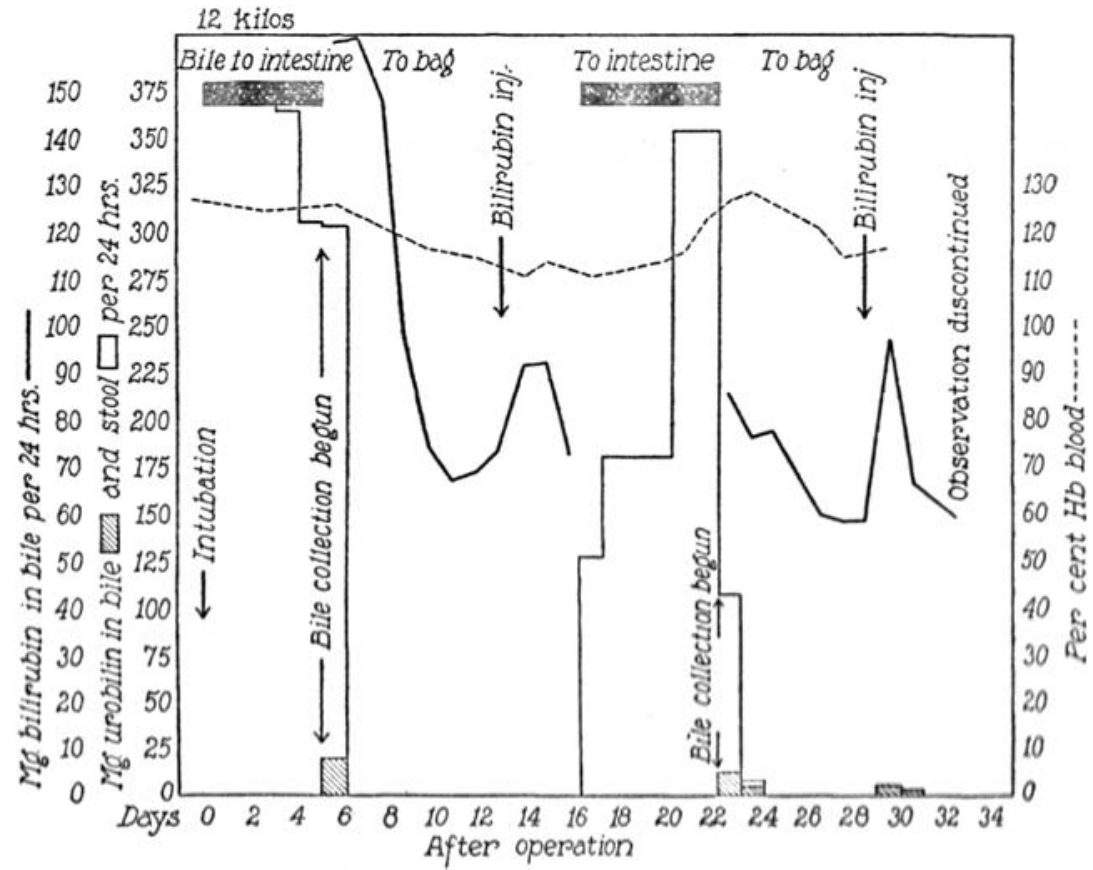

TExT-Fig. 9. Effect of introducing pure bilirubin into the duodenum of an animal with an "altercursive intubation."

A notable rise in the bilirubin output followed the injections, on both the occasions upon which they were practiced. The other findings are discussed in the text.

the solution of purified bilirubin was given in divided portions through the efferent tube into the duodenum. This was on the 13th day. The animal's bile of the 14th day contained $90.0 \mathrm{mg}$. of bilirubin and that of the next 24 hour period 90.6, with a return to $71.2 \mathrm{mg}$., a normal figure, 24 hours later. No urobilin. appeared in the bile following the bilirubin administration. The finding was unique in this respect. Bile collection was now stopped, and the secretion allowed to reach the intestine as previously. After an interval of 1 week the bile was again 
diverted to the collecting balloon, and in due course the experiment was repeated, using $100 \mathrm{mg}$. of pure bilirubin. For the 3 days prior to the administration of the pigment the bile had contained $58.9,57.4$, and $57.7 \mathrm{mg}$. of bilirubin in each 24 hours. The amount on the day following the injections rose to $95.0 \mathrm{mg}$. of bilirubin, an increase of 65 per cent. It fell in the next 24 hours to $65.2 \mathrm{mg}$., almost a normal value, and 2 days later it approximated this closely. Urobilin appeared in the bile following the pigment administration. It had been found too for a brief period immediately after bile collection was begun, just as previously.

Similar findings were obtained in two other animals, that is to say, in these there was not only a rise in the bilirubin output in the bile but a reappearance of urobilin in it as well, after each injection of pure bilirubin into the duodenum.

\section{The Effect of Urobilin Feedings.}

The feeding experiments were repeated with purified urobilin. Evidence had been obtained in our previous work ${ }^{2}$ that this pigment, as well as bilirubin, is absorbed. The findings were not as striking as those with bilirubin, as was to have been expected since much of the pigment is probably reduced in the intestine to unrecognizable forms. The results, however, were definite enough to demonstrate an absorption of it clearly.

For administration by stomach tube 100 to $500 \mathrm{mg}$. of the purified pigment were taken up in $50 \mathrm{cc}$. of 1 per cent ammonium hydroxide and neutralized. Six such experiments in all were performed, two with each of three different dogs, intubated for total bile deprivation. The bile, feces, and urine of these dogs were free from urobilin before the feedings. Each animal, on the day after, showed urobilin in the bile and at times continued to show it for another 48 hours. In some instances there was a slight increase in the bilirubin output from the liver after the feedings, suggesting that perhaps part of the resorbed urobilin was converted to bilirubin.

In one instance a $16 \frac{3}{4} \mathrm{kilo} \mathrm{dog}$, fed $250 \mathrm{mg}$. of the pigment on 2 successive days, urobilin appeared not only in the bile and stool but in the urine as well, and the bilirubin of the bile was increased. Since the findings were artificial they have not been charted.

Specimen protocols follow (Text-figs. 10 and 11).

Dog A (Text-fig. 10), 9 kilos. Cannulation of the common duct was accomplished as usual under ether anesthesia. The animal remained in excellent condi- 
PHILIP D. MCMASTER AND ROBERT ELMAN

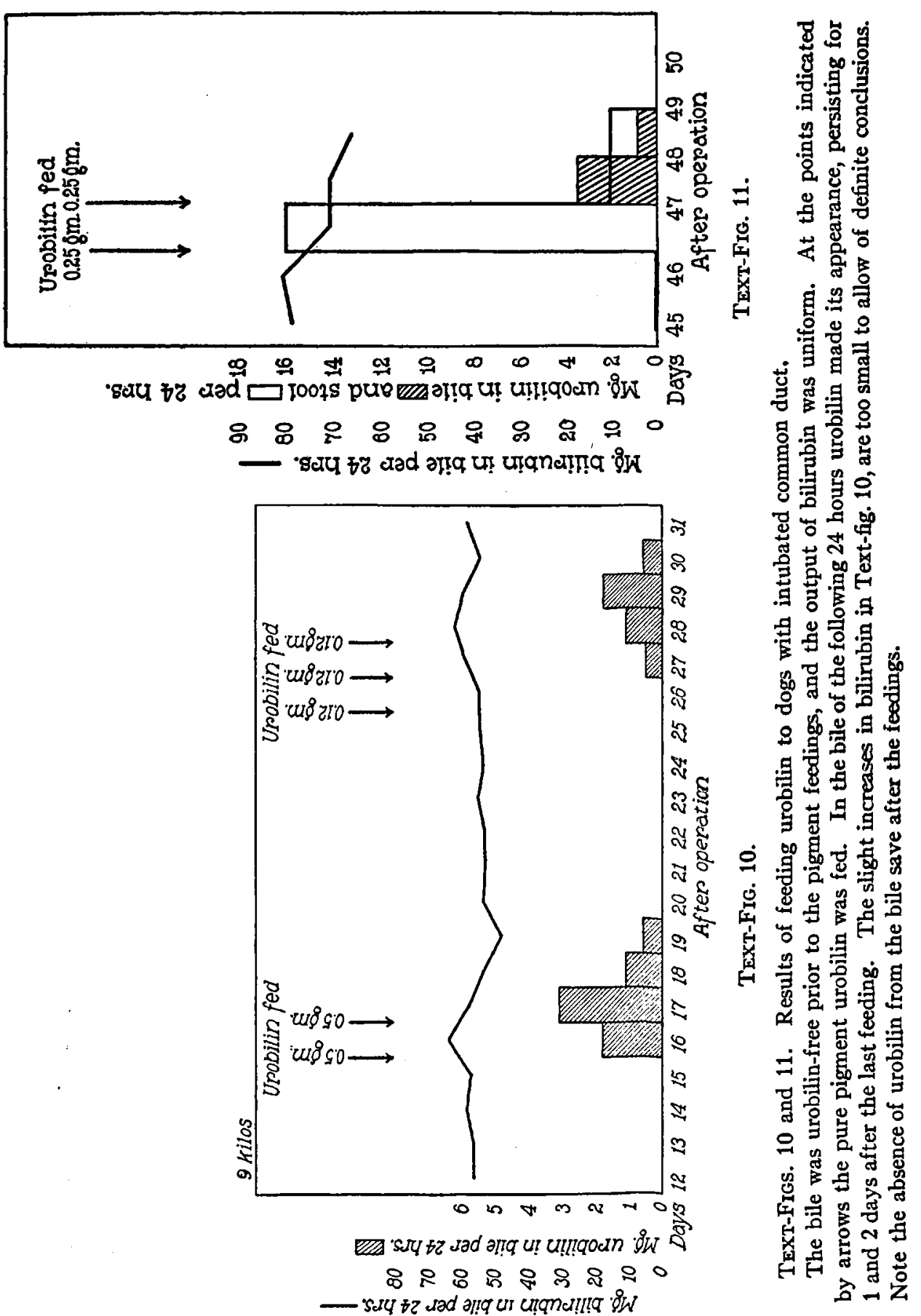


tion and was kept on the standard diet of bread, milk, and lean meat. For 2 weeks no urobilin was present in bile, feces, or urine, and the output of bilirubin in the bile became uniform after the usual postoperative changes. On the 15th day $0.5 \mathrm{gm}$. of urobilin in solution was fed by mouth.

The hepatic bile of the 24 hours following the feeding contained much urobilin and there occurred a slight increase in the bile pigment. Another feeding of 0.5 gm. of urobilin was given with result that it doubled in quantity in the bile. A week later, when the bile had again become urobilin-free, the pigment was again given by mouth in weak alkaline, aqueous solution, on several successive days, to the amount each time of $120 \mathrm{mg}$. The pigment promptly reappeared in the bile and persisted there for 3 days after the feedings had been stopped.

Dog E (Text-fig. 11), 16 kilos. Common duct intubation as usual under ether anesthesia. During 46 days thereafter the animal was in excellent condition and no urobilin was present in bile, feces, or urine. $250 \mathrm{mg}$. of urobilin were now given in neutralized ammoniacal solution by mouth. The following day urobilin appeared in the urine and stool but not in the bile. Another feeding of it was given, and for $\mathbf{2}$ days thereafter the bile was found to contain it to the amounts of 4.0 and $1.0 \mathrm{mg}$. respectively in each 24 hour period.

The results in the third animal will not be charted since they were in all ways. similar to those described.

The changes, though not as pronounced as those occurring on bilirubin administration, show that feedings of pure urobilin to intubated dogs yielding bile free from the pigment, result in its presence in the secretion.

\section{DISCUSSION.}

The experiments here reported supply direct proof that both bilirubin and urobilin are absorbed from the intestine. In previous papers from this laboratory it was shown that, after bile feedings, much more bilirubin is excreted into the bile ${ }^{1,2}$ but the possibility that the increase is due to hemolysis from absorbed bile salts could not be wholly ruled out. This has now been accomplished. Feedings of sodium glycocholate as such, even in large amounts, cause no change in the bilirubin output, though greatly increasing the bile quantity. In one of our previous papers ${ }^{2}$ it has been shown that bile feedings cause an increased bilirubin and urobilin output by animals with a "sampling intubation;" and when they are given to animals losing all of the secretion into a collecting balloon cause the latter pigment to reappear in the bile so obtained as well as an in- 
creased bilirubin yield. It has remained to determine whether feedings of bilirubin by mouth, or direct introduction of it into the duodenum, bring about the same effects or further whether they follow upon feedings of purified urobilin.

This has now been done. The findings leave no alternative to the conclusion that an enterohepatic circulation of bile pigments exists under normal circumstances. That this is true of bile salts has long been known, and that it holds true for cholesterol has recently been shown in this laboratory.15 Bile is treated by the organism as though it were anything but a waste product. But can one assume that the physiological mechanism for the conservation and reutilization of its major ingredients is purposeful in character? The bile salts have well known uses; and evidence has been presented ${ }^{18}$ that bilirubin is conserved in porportion to the need for hemoglobin from which it is primarily derived. The amount of cholesterol put out in the bile, which might, at the worst, be lost therewith, is negligible; and the absorption of it that takes place from the gut may be merely one instance of a general phenomenon. One can only conjecture as concerns a possible use of urobilin.

The fact should be emphasized here that our observations were made upon healthy, robust animals. In succeeding papers the findings after liver injury and blood destruction will be described. The enterohepatic circulation of bilirubin and urobilin is of special practical interest in such connection.

The phenomenon of pigment reabsorption, that we have demonstrated, would appear to be far reaching in its implications. It must be taken into account in any hypothesis of pigment metabolism. The clinical utilization of the urobilin output in the feces as a measure of blood destruction should only be undertaken with the understanding that urobilin is subject, not only to degradation but to reabsorption.

SUMMARY.

Direct proof of the absorption of bile pigments from the intestinal tract is presented.

${ }^{16}$ Broun, G. O., McMaster, P. D., and Rous, P., J. Exp. Med., 1923, xxxvii, 733. 
Feedings of pure bilirubin by mouth to dogs, like those of whole bile, increase greatly the amount of this pigment in the hepatic bile. Control feedings of bile salts, while greatly increasing the bile quantity, cause no change in the pigment output. Instillations of pure bilirubin directly into the duodenum of dogs, appropriately intubated, are followed by pronounced increases in the bilirubin output in the bile.

Feedings of pure urobilin to dogs, yielding urobilin-free bile, as result of diversion of the secretion from the intestine, are followed by the appearance of this pigment in the bile. 